\title{
Head and neck reconstruction in the vessel depleted neck
}

\author{
Andrew D. P. Prince ${ }^{1}$, Michael T. Broderick ${ }^{1}$, Molly E. Heft Neal $^{2}$, Matthew E. Spector ${ }^{2}$ \\ ${ }^{1}$ University of Michigan Medical School, Ann Arbor, MI, USA; ${ }^{2}$ Department of Otolaryngology-Head and Neck Surgery, University of Michigan \\ Health System, Ann Arbor, MI, USA \\ Contributions: (I) Conception and design: All authors; (II) Administrative support: All authors; (III) Provision of study materials or patients: All \\ authors; (IV) Collection and assembly of data: All authors; (V) Data analysis and interpretation: All authors; (VI) Manuscript writing: All authors; (VII) \\ Final approval of manuscript: All authors. \\ Correspondence to: Matthew E. Spector, MD, FACS. Department of Otolaryngology-Head and Neck Surgery, University of Michigan Health System, \\ 1500 E. Medical Center Dr., 1904 Taubman Health Center, SPC 5312, Ann Arbor, MI 48109-5312, USA. Email: mspector@med.umich.edu.
}

\begin{abstract}
Microvascular free tissue transfer has revolutionized reconstruction and subsequently functional outcomes in the head and neck, but requires suitable recipient vessels for successful results. Recipient vessels can be significantly compromised by prior surgery, radiation therapy, or existing and/or underlying vascular disease in the neck. When further microvascular reconstruction is required in the vessel-depleted neck, identification of appropriate vessels for anastomosis can be difficult and can present complex decisions for the surgeon as well as the patient. In this article, we review the available literature on the vessel depleted neck $(\mathrm{VDN})$ and the possible vessel options. We present critical strategies for preoperative treatment planning and vessel selection in these patients. We also discuss the benefits and limitations of arterial and venous options while commenting on our unique institution's experiences. The external carotid branches as well as the available subclavian artery branches are presented in detail. The venous anatomy is also described, with particular focus on the accompanying veins and cephalic vein. We provide guidance on the selection and modification of free flaps to achieve the greatest function and cosmetic outcomes in the VDN. Our collection of advanced management techniques will provide surgeons with more options to manage the complexity of the VDN, and to further help patients understand the risk and benefits of these selections.
\end{abstract}

Keywords: Head and neck cancer; microvascular reconstruction; vessel depleted neck (VDN); arterial anastomosis

Received: 02 July 2020; Accepted: 30 July 2020; Published: 03 August 2020.

doi: $10.21037 /$ fomm-20-38

View this article at: http://dx.doi.org/10.21037/fomm-20-38

\section{Introduction}

Microvascular free flap reconstruction is a cornerstone in the management of head and neck cancer. Microsurgical therapy has been evolving for over 70 years and has shown excellent success with flap survival rates greater than $95 \%$ $(1,2)$. An important determinant of success in head and neck free tissue transfer is the quality of the free flap pedicle and the recipient vessels used for microvascular anastomosis. Challenging reconstructive cases are posed by previously treated necks with either surgery, radiation with or without chemotherapy, or a combination of both. Patients with these prior interventions have cervical anatomy with distorted tissue planes, inflammation, and scarred vessels leaving a patient's neck depleted of suitable recipient vessels. A vessel depleted neck (VDN) occurs in around $7 \%$ of all patients receiving microvascular reconstructions in the head and neck (3). Frequently, transplants of remote vessels are sought from undamaged areas. A vast diversity in the vessels and transplants suitable for VDN reconstruction exist and management selection can be challenging. Unfortunately, broad evidence-based approaches are limited and individual surgeon experiences dominate the literature (4). We set forth to collect the available techniques and experimental and/or anecdotal solutions within the literature. In our review, we evaluate microvascular reconstruction in the VDN and provide approaches to manage these patients who require advanced reconstruction. 


\section{Preoperative planning}

A detailed preoperative assessment should be conducted before any operation and is of particular importance in previously treated patients. A complete history is essential and should ascertain comorbidities and nutrition status that give insight into neck vessel health and tissue healing capacity. This should include a history of claudication symptoms, as a number of patients have concurrent atherosclerosis from smoking. A detailed review of previous interventions and treatments of the neck is important including surgeries, complications, and radiation. Equally important, a detailed clinical examination will elicit evidence of postradiotherapy changes of the neck, scars from previous operations, potential patency of external jugular veins bilaterally, and evidence of previous operations that might influence vessel or flap selection. A shared decisionmaking approach between provider and patient is critical in developing a plan of care. Not all patients are appropriate candidates for surgery and alternative therapies should be considered after a discussion of the risks and benefits. This is especially important in patients undergoing secondary reconstructive surgery without cancer recurrence, as this elective surgery could be deferred for alternative treatment options.

When approaching a VDN it is important to first consider which recipient vessels may still be present and usable, based on the prior history, imaging, and physical exam. Recipient vessel selection can be impacted by many factors including, defect location, vessel anatomic variations, quality of the available recipient vessels, and anastomotic method $(5,6)$. Each one of these can have a substantial impact on the operation and outcome (5). Ideally, the recipient artery should be scar and disease free, of suitable length, and have a similar caliber to the donor vessel (7). Algorithms for vessel selection in the literature are based on several features, including ease of dissection, historical reliability, vessel caliber, and proximity to the defect $(7,8)$. The next important decision is considering which particular free flap should be harvested. Flap selection for appropriate characteristics, pedicle size, and length is important, but must also match the available recipient vessels. Choosing a flap with a long vascular pedicle can alleviate some of the difficulties associated with reaching remote recipient vessels, but if it is necessary to harvest a flap with a short vascular pedicle then the surgeon must consider using vein grafts. This decision is dependent on surgeon comfort and training, and requires an important discussion with the patient regarding the expected functional outcome with a particular flap, weighed against the degree of difficulty and risk of flap failure.

Preoperative imaging of the VDN is equally important to proper operative planning. Imaging in the form of a computed tomography (CT) or magnetic resonance imaging (MRI) with contrast can provide useful to confirm vessel patency and to provide anatomic positioning after prior neck manipulation. A dual phase CT angiogram is ideal for highlighting arterial and venous vasculature, often times the most important planning step (9). Single phase CT and MRI angiography are good alternatives that demonstrate arterial flow but omit high quality venous vasculature views. With a dual phase CT angiogram from the skull base to the diaphragm, patency of all important vessels can be assessed (10). While imaging is a useful adjunct, it does not replace intraoperative evaluation of the recipient vessel's condition.

\section{Arteries}

Arterial options are varied in the VDN and appropriate selection for each surgical case can be challenging. In Table 1, we present the relative strengths and weaknesses of a collection of arteries and veins trusted in the VDN. We further expand on the reconstructive techniques and utility of the vessels in the subsequent section.

\section{External carotid artery (ECA)}

In the normal neck the ECA branches are trusted and well used for anastomosis. The ECA gives off six branches before terminating as the maxillary and superficial temporal arteries. These include the superior thyroid, lingual, and facial arteries (anterior branches), the ascending pharyngeal (medial branch) and the occipital and posterior auricular (posterior branches). The facial artery, the superior thyroid artery, superficial temporal artery, and the lingual artery are often favored due to their consistent anatomic location, length, diameter, and perfusion.

The branches of the ECA are often considered the gold standard but in the VDN their utility is often limited. The majority of previous surgery and radiation fields include these vessels, making them heavily scarred and extremely difficult to use. In addition, their location under the mandible, and a patient's limited neck extension due to radiation changes, makes surgical access challenging. The most utilized branch of the ECA in the VDN is the 
Table 1 A collection of donor vessel strengths and weaknesses

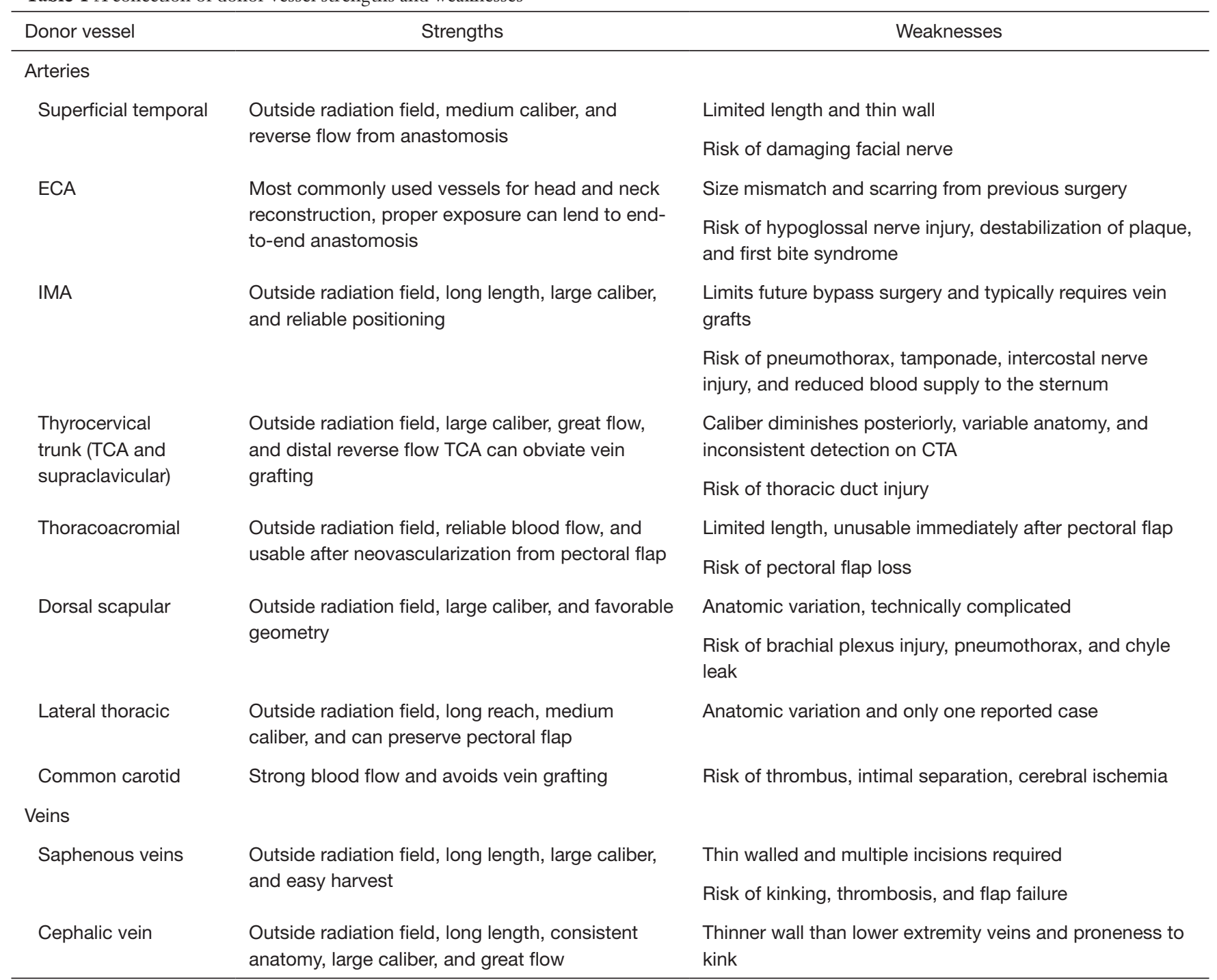

ECA, external carotid artery; IMA, internal mammary artery; TCA, transverse cervical artery.

superficial temporal artery used $14.9 \%$ of the time as it often lies outside the field of radiation, Figure 1 (4). The superficial temporal artery is found in the preauricular area and is in continuity with the ophthalmic artery and occipital artery in the forehead. This anastomosis provides excellent reverse flow even if the ECA has been sacrificed in prior operations (10). It is a reliable option but limited by its length, risk of facial nerve injury, thin wall, and predisposition to kinking $(10,11)$.

When branches of the ECA are unavailable, ECA transposition and end-to-end anastomosis is a potential option for arterial inflow into the free flap pedicle (12).
Oftentimes the ECA has been exposed in level I and II neck dissections leading to an easier approach and less incisions. Ligation of the vessel distally provides greater recipient vessel length and potentially simpler anastomosis outside of the cervico-mandibular angle (12). This exposure may enable end-to-end anastomosis rather than a more complex end-to-side coupling. Limitations include size mismatch and dangerous collateral blood depletion to the brain, especially in at-risk patients with stenotic vessels or previous stroke history (13). Hypoglossal nerve injury, potential destabilization of plaque, and first bite syndrome are also possible complications to consider $(14,15)$. 


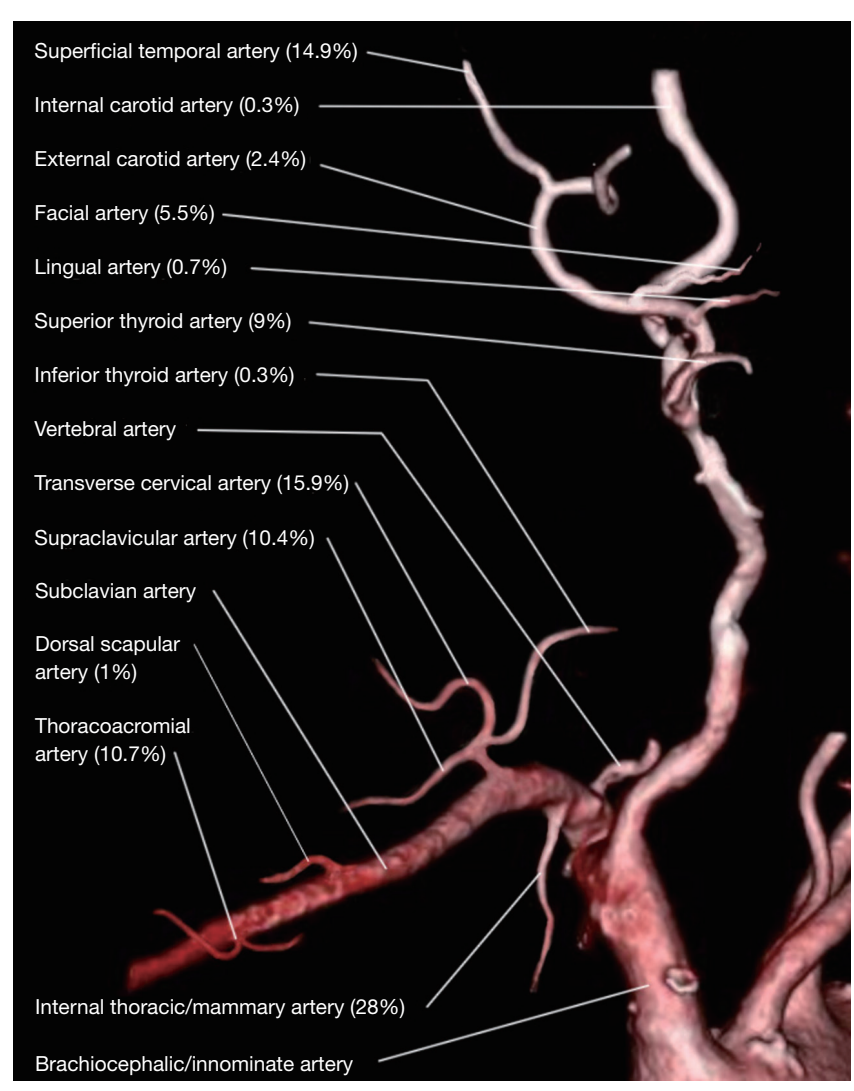

Figure 1 Most utilized arterial options in the vessels depleted neck. This computerized tomography angiogram of the neck presents arterial options, labeled with percentages of how commonly they are used, for microvascular anastomosis in the vessel depleted neck. This figure does not include the radial artery and a pedicle of a previous flap which together contribute $0.6 \%$ to the total percentage. Percentages are reported from reference (4).

\section{Internal mammary artery (IMA)}

The IMA originates from the subclavian artery and the second to third intercostal space is the most reliable location for vessel harvest. The right IMA is considered to be the most reliable of all arterial anastomosis options in the VDN (4), with a long pedicle, large caliber, and reliable vessel-positioning $(3,16)$. There are two primary methods for harvesting the IMA and internal mammary vein (IMV); the traditional method, or the less often used rib sparing technique (16). The rib-sparing technique's primary role is in breast reconstruction, but it can be used in the head and neck when there is a large flap pedicle or less recipient vessel length is needed. Surgeons trained in plastic surgery-based head and neck fellowships may be more comfortable with these vessels given their common use in this specialty. The main disadvantages include potential for pneumothorax, intercostal nerve injury, cosmetic deformity of the donor site, and reduced blood supply to the sternum $(3,17)$. Furthermore, cardiac tamponade is a rarely reported complication, and possible future bypass surgery with an internal mammary graft is a contraindication for vessel dissection $(3,18)$.

\section{Thyrocervical trunk: transverse cervical, supraclavicular, inferior thyroid}

Branching from the subclavian artery, the thyrocervical trunk offers additional advanced alternatives. The commonly used transverse cervical artery (TCA) and supraclavicular artery often branch from the thyrocervical trunk in the base of level IV. These vessels have an ideal longitudinal orientation and provide great flow with a large caliber (19). In addition, they are often spared under the fascia of a level 4 neck dissection, out of the previous operative field. The caliber of the TCA is largest closest to the origin and greatly diminishes as it's followed posteriorly, often requiring a long pedicle flap, or interposition vein graft to anastomose (3). However, the distal reverse flow TCA has been found to be a reliable recipient option that obviates the need for long vein grafts to reach distant recipient vessels when other common alternatives are unavailable (20). Other challenges include variable anatomy, inconsistent detection on CT angiogram, and possible thoracic duct injuries (4).

\section{Thoracoacromial artery}

There remains a paucity of literature on the use of the thoracoacromial vessels; however, this vessel is typically undissected and available for head and neck reconstruction, in the absence of previous pectoralis flap reconstruction. The vessel classically divides into four branches: the pectoral, deltoid, clavicular, and acromial arteries (21). The thoracoacromial venous system generally mirrors the arterial branching. The dissection of the thoracoacromial system is explained elsewhere (22), but the branches can usually be viewed after a horizontal incision through the pectoralis muscle and careful tissue plane dissection. The branches of the system can be dissected distally to achieve transposition of the artery over the clavicle and into the neck (22). The trunk provides reliable blood flow and is 
often usable after radiation therapy and a previous pectoral flap harvest $(22,23)$.

The substantial drawback of this technique is the potential loss of the major vascular pedicle to the pectoralis major flap. However, it is possible to use another branch of the thoracoacromial artery for anastomosis and the pectoral branch can be preserved. Other limitations include unsuitability for anastomosis if a pedicle flap has been raised shortly before, and the possibility of needing an interposition vein graft except when using a long vascular pedicle (3).

\section{Dorsal scapular artery (DSA)}

Scarce literature exists on the DSA use in the VDN, but our institution has previously published on the method of dissection, and has found them to be reliable and an invaluable technique (24). Major advantages of the vessel include its distance from usual treatment fields, large caliber, and favorable geometry. In most cases the DSA branches from the subclavian artery and travels through the posterior triangle. It is important to note, the DSA can be found branching from the TCA in almost $1 / 3$ of cases and there is a large degree of anatomic variation in relation to the brachial plexus (25). Care must be taken around the brachial plexus and subclavian artery, as these can be exposed in the dissection. A Doppler has been utilized by our group in cases of difficult dissection as the DSA can usually be found just superior to the superior trunk of the brachial plexus. The clavicle can make the exposure difficult. The greatest limitation is the low anatomic location of the vessel requiring a long vascular pedicle or vein graft, although with careful dissection, the vessel can be traced to its insertion to the scapula and reflected superiorly into the neck (24). Other potential risks, our particular institution has not experienced, include pneumothorax and chyle leak.

\section{Lateral thoracic artery (LTA)}

The LTA is an option as a recipient vessel for head and neck free flap microvascular reconstruction, but is reported in only one case in the literature to our knowledge (24). The vessel is often well situated outside the radiation field and can offer greater reach and caliber than the thoracoacromial system. Furthermore, it is rather simple to harvest and may allow for preservation of the pectoral branch of thoracoacromial artery, preserving the pectoralis major flap. The LTA anatomic origin is usually a branch of the second part of the axillary artery but has been noted to have variable anatomic origins (26). Greater studies are essential before determining its true utility when it comes to reach, diameter, blood flow, and risk to potential pectoralis major flaps.

\section{Common carotid artery}

A less common but viable technique in the VDN is using the common carotid artery. If other common vessels are unavailable the carotid end-to-side anastomosis allows for strong perfusion without the need for a vein graft (27). This technique is hazardous and can lead to cerebral ischemia, but has been proven viable as long as there is well-developed collateral circulation through a patent ECA to maintain internal carotid perfusion (28). Furthermore, one study demonstrated the use of a Pruitt-Inhara shunt to maintain perfusion of the internal carotid artery when the ECA was otherwise compromised, greatly reducing risk (27). Regardless of potential, end-to-side technique with the common carotid is often avoided due to carotid atherosclerotic plaque buildup increasing risk of thrombus and intimal separation (8).

\section{Veins}

Adequate venous drainage is critical in the VDN and the easiest option is to harvest the accompanying veins of the selected recipient artery branches, as often practiced with the mammary vessels, the thoracoacromial vessels, and thyrocervical trunk vessels. The internal and external jugular veins are also major venous systems used for anastomosis, but can be unavailable in the VDN (10). The surgeon can also consider the use of the contralateral neck if the flap pedicle is long and the anastomosis won't be under tension. It is often tempting to consider using the anterior jugular veins, but many patients undergoing head and neck reconstructive surgery will have or have had a tracheostomy. Unfortunately, iatrogenic injury to the anterior jugular veins during a tracheotomy or the inflammation associated with the stoma make these veins very unfavorable.

Veins are also a commonly used tool in reconstruction to lengthen the reach of free flap pedicles. If it is necessary to harvest a flap with a short vascular pedicle then vein grafts should be prepared prior to free flap harvesting. The greater and lesser saphenous veins are regularly utilized for their length, large-caliber, and easy harvest. Surgeons should carefully consider the vessel options as vein grafts 
require two anastomotic sites, have more risk of kinking, thrombosis, and have been reported to increase risk of flap failure by nearly $24 \%$ (29).

\section{Cephalic vein}

The cephalic vein provides an excellent alternative to the internal and external venous system. While the dissection technique is described elsewhere (30), the vein is useful in the VDN as it lies external to any prior surgical or radiation field. The cephalic vein has been deemed the "lifeboat" vessel for its extensive advantages including consistent anatomy, long pedicle, great flow and caliber, and low risk of thrombosis $(4,31)$. The cephalic vein is preferred almost $25 \%$ of the time (4), because of its simple dissection and its extensive length allows for transposition over the clavicle to reach almost any area of the ipsilateral neck. The main limitations of this vein are its thin wall compared to the lower extremity veins and its proneness to kink when crossing the clavicle (3).

In the VDN when suitable vessels are less readily available the cephalic vein has been used in a corlett loop (32). The vein is first anastomosed to the ECA; it then can be divided and used in a single or double staged procedure (33). The proximal end is attached to the vein of the flap, while the distal end, still connected to the ECA, is anastomosed to the flap artery (32). There is a higher rate of failure in two-stage arteriovenous fistulas (33), and to date the corlett loop has only been completed in single-stage procedures in the head and neck (32). There is a risk of twisting of the vein and at our institution we avoid the two stage corlett loop due to the scarring that can occur between the first and second stage, as well as the risk of heart failure due to creation of a large vessel arteriovenous fistula.

\section{Free flap choice}

Having a wide armamentarium of free microvascular flaps allows surgeons to tackle the challenges of the VDN. After the available vessels have been determined, there are hopefully several free flap options available that can provide similar function and cosmetic outcomes. However, in the VDN the most important criteria are based on reliability, the reconstructive indication, and the defect configuration (4). More weight may be put on a bulkier well-vascularized flap to avoid fistula, wound healing concerns, and surgical reoperation.
The versatility of the free flap in the VDN can be greatly enhanced when it contains a longer pedicle length allowing for easier anastomosis with distance recipient vessels. The most commonly used include the perforator based anterolateral thigh flap and the radial forearm free flap (RFFF) which often provide the needed length and characteristics suitable to the VDN (4). Likewise, the osseous flaps that combine functionality with the longest pedicles are used significantly in the VDN including the fibula and scapula free flaps (4).

Modifying an existing flap to provide the needed length has also been demonstrated in the literature. One technique is to extend the scapula free flap pedicle by harvesting the thoracodorsal artery and using the distal end of the thoracodorsal artery as the anastomosis (3). This creates a reverse flow in the blood to the circumflex scapular artery supplying the flap. However, a vein graft might still be needed for the venous anastomosis and this technique eliminates the future use of a latissimus dorsi free flap (3).

Another viable technique when other options are unavailable includes the use of the RFFF to create a vascular bridge or harvest of the radial artery to use for arterial anastomosis (34). In many of the available VDN techniques recipient sites often require a vascular conduit to bridge the distance to the flap pedicle. While the RFFF vascular pedicle supplies the second flap, the soft tissue of the flap can contribute to the reconstruction of the surgical defect. Both interposition vein grafts and arterio-venous loops have been used as vascular bridges but pose heavy risks (33). Using a reliable RFFF as a vascular bridge may achieve greater flap survival and fewer safety concerns. The possible drawbacks include greater harvesting time for two free flaps and loss of two flaps in the setting of thrombosis of the RFFF pedicle (34).

\section{Conclusions}

The VDN is highly challenging in cases of advanced reconstruction. Meticulous surgical planning and creative solutions are often necessary to solve these complex cases. Detailed anatomic knowledge and an understanding of the benefits and limitations of each arterial and venous option are critical. Minimal treatment evidence in the VDN exists and reported techniques are limited to individual surgeon experiences. This review outlines the myriad of proven strategies and creative solutions in an effort to guide individualized solutions for every patient. 


\section{Acknowledgments}

The authors would like to acknowledge Eric Wizauer for critical assistance with image procurement and figure development.

Funding: MEHN was funded by the NIH (T32 DC005356).

\section{Footnote}

Provenance and Peer Review: This article was commissioned by the Guest Editor (Paolo Cariati) for the series "Microvascular reconstruction of head and neck oncological defects-state of the art" published in Frontiers of Oral and Maxillofacial Medicine. The article has undergone external peer review.

Conflicts of Interest: All authors have completed the ICMJE uniform disclosure form (available at: https://fomm. amegroups.com/article/view/10.21037/fomm-20-38/coif). The series "Microvascular reconstruction of head and neck oncological defects—state of the art" was commissioned by the editorial office without any funding or sponsorship. The authors have no other conflicts of interest to declare.

Ethical Statement: The authors are accountable for all aspects of the work in ensuring that questions related to the accuracy or integrity of any part of the work are appropriately investigated and resolved.

Open Access Statement: This is an Open Access article distributed in accordance with the Creative Commons Attribution-NonCommercial-NoDerivs 4.0 International License (CC BY-NC-ND 4.0), which permits the noncommercial replication and distribution of the article with the strict proviso that no changes or edits are made and the original work is properly cited (including links to both the formal publication through the relevant DOI and the license). See: https://creativecommons.org/licenses/by-nc-nd/4.0/.

\section{References}

1. Khouri RK, Cooley BC, Kunselman AR, et al. A prospective study of microvascular free-flap surgery and outcome. Plast Reconstr Surg 1998;102:711-21.

2. Suh JD, Sercarz JA, Abemayor E, et al. Analysis of outcome and complications in 400 cases of microvascular head and neck reconstruction. Arch Otolaryngol Head Neck Surg 2004;130:962-6.
3. Jacobson AS, Eloy JA, Park E, et al. Vessel-depleted neck: techniques for achieving microvascular reconstruction. Head Neck 2008;30:201-7.

4. Frohwitter G, Rau A, Kesting MR, et al. Microvascular reconstruction in the vessel depleted neck - A systematic review. J Craniomaxillofac Surg 2018;46:1652-8.

5. Tan BK, Wong CH, Chen HC. Anatomic variations in head and neck reconstruction. Semin Plast Surg 2010;24:155-70.

6. Nahabedian MY, Singh N, Deune EG, et al. Recipient vessel analysis for microvascular reconstruction of the head and neck. Ann Plast Surg 2004;52:148-55; discussion 56-7.

7. Yazar S. Selection of recipient vessels in microsurgical free tissue reconstruction of head and neck defects. Microsurgery 2007;27:588-94.

8. Chia HL, Wong CH, Tan BK, et al. An algorithm for recipient vessel selection in microsurgical head and neck reconstruction. J Reconstr Microsurg 2011;27:47-56.

9. Du E, Patel S, Huang B, et al. Dual-phase CT angiography for presurgical planning in patients with vessel-depleted neck. Head Neck 2019;41:2929-36.

10. Breik O, Praveen P, Parmar S. The vessel-depleted neck in head and neck microvascular reconstruction: extreme solutions for extreme situations. Curr Opin Otolaryngol Head Neck Surg 2020;28:129-35.

11. Wong KK, Higgins KM, Enepekides DJ. Microvascular reconstruction in the vessel-depleted neck. Curr Opin Otolaryngol Head Neck Surg 2010;18:223-6.

12. Garg RK, Poore SO, Wieland AM, et al. Recipient vessel selection in the difficult neck: Outcomes of external carotid artery transposition and end-to-end microvascular anastomosis. Microsurgery 2017;37:96-100.

13. Dalainas I, Avgerinos ED, Daskalopoulos ME, et al. The critical role of the external carotid artery in cerebral perfusion of patients with total occlusion of the internal carotid artery. Int Angiol 2012;31:16-21.

14. Kim T, Chung S, Lanzino G. Carotid artery-hypoglossal nerve relationships in the neck: an anatomical work. Neurol Res 2009;31:895-9.

15. Laccourreye O, Werner A, Garcia D, et al. First bite syndrome. Eur Ann Otorhinolaryngol Head Neck Dis 2013;130:269-73.

16. Roche NA, Houtmeyers P, Vermeersch HF, et al. The role of the internal mammary vessels as recipient vessels in secondary and tertiary head and neck reconstruction. J Plast Reconstr Aesthet Surg 2012;65:885-92.

17. Carrier M, Grégoire J, Tronc F, et al. Effect of internal 
mammary artery dissection on sternal vascularization. Ann Thorac Surg 1992;53:115-9.

18. Nahabedian MY. The internal mammary artery and vein as recipient vessels for microvascular breast reconstruction: are we burning a future bridge? Ann Plast Surg 2004;53:311-6.

19. Urken ML, Vickery C, Weinberg H, et al. Geometry of the vascular pedicle in free tissue transfers to the head and neck. Arch Otolaryngol Head Neck Surg 1989;115:954-60.

20. Ciudad P, Agko M, Manrique OJ, et al. The retrograde transverse cervical artery as a recipient vessel for free tissue transfer in complex head and neck reconstruction with a vessel-depleted neck. Microsurgery 2017;37:902-9.

21. Reid CD, Taylor GI. The vascular territory of the acromiothoracic axis. Br J Plast Surg 1984;37:194-212.

22. Harris JR, Lueg E, Genden E, et al. The thoracoacromial/ cephalic vascular system for microvascular anastomoses in the vessel-depleted neck. Arch Otolaryngol Head Neck Surg 2002;128:319-23.

23. Aycock JK, Stenson KM, Gottlieb LJ. The thoracoacromial trunk: alternative recipient vessels in reoperative head and neck reconstructive microsurgery. Plast Reconstr Surg 2008;121:88-94.

24. Rosko AJ, Ryan JT, Wizauer EJ, et al. Dorsal scapular artery as a recipient vessel in the vessel-depleted neck during free tissue transfer in head and neck reconstruction. Head Neck 2017;39:E72-6.

25. Ikka L, Mihalea C, Achour NB, et al. The origin of the dorsal scapular artery: anatomic variations and surgical applications. Surg Radiol Anat 2016;38:1021-7.

doi: 10.21037/fomm-20-38

Cite this article as: Prince ADP, Broderick MT, Heft Neal ME, Spector ME. Head and neck reconstruction in the vessel depleted neck. Front Oral Maxillofac Med 2020;2:13.
26. Loukas M, du Plessis M, Owens DG, et al. The lateral thoracic artery revisited. Surg Radiol Anat 2014;36:543-9.

27. Salgarello M, Snider F, Finocchi V, et al. The PruittInahara carotid shunt as an assisting tool to anastomose the arterial free flap pedicle to the internal carotid artery in the vessel-depleted neck. Microsurgery 2011;31:234-6.

28. Lee HS, Park SY, Jang HJ, et al. Free jejunal graft for esophageal reconstruction using end-to-side vascular anastomosis and extended pharyngo-jejunostomy. Ann Thorac Surg 2012;93:1850-4.

29. Nelson JA, Fischer JP, Grover R, et al. Vein grafting your way out of trouble: Examining the utility and efficacy of vein grafts in microsurgery. J Plast Reconstr Aesthet Surg 2015;68:830-6.

30. Vasilakis V, Patel HD, Chen HC. Head and neck reconstruction using cephalic vein transposition in the vessel-depleted neck. Microsurgery 2009;29:598-602.

31. Horng SY, Chen MT. Reversed cephalic vein: a lifeboat in head and neck free-flap reconstruction. Plast Reconstr Surg 1993;92:752-3.

32. Ethunandan M, Cole R, Flood TR. Corlett loop for microvascular reconstruction in a neck depleted of vessels. Br J Oral Maxillofac Surg 2007;45:493-5.

33. Lin CH, Mardini S, Lin YT, et al. Sixty-five clinical cases of free tissue transfer using long arteriovenous fistulas or vein grafts. J Trauma 2004;56:1107-17.

34. Ciudad P, Agko M, Date S, et al. The radial forearm free flap as a "vascular bridge" for secondary microsurgical head and neck reconstruction in a vessel-depleted neck. Microsurgery 2018;38:651-8. 